\title{
$\mathrm{g}^{\circ}$
}

\section{Carbon Nanotubes as Cooper-Pair Beam Splitters}

\author{
L. G. Herrmann, ${ }^{1,2,5}$ F. Portier, ${ }^{3}$ P. Roche, ${ }^{3}$ A. Levy Yeyati, ${ }^{4}$ T. Kontos, ${ }^{1,2, *}$ and C. Strunk ${ }^{5}$ \\ ${ }^{1}$ Ecole Normale Supérieure, Laboratoire Pierre Aigrain, 24, rue Lhomond, 75231 Paris Cedex 05, France \\ ${ }^{2}$ CNRS, UMR 8551, Laboratoire associé aux universités Pierre et Marie Curie et Denis Diderot, Paris, France \\ ${ }^{3}$ Service de physique de l'état Condensé, CEA, 91192 Gif-sur-Yvette, France. \\ ${ }^{4}$ Departamento de Física Teórica de la Materia Condensada C-V, Universidad Autónoma de Madrid, E-28049 Madrid, Spain \\ ${ }^{5}$ Institut für experimentelle und angewandte Physik, Universität Regensburg, Universitätsstr.31, 93040 Regensburg, Germany
}

(Received 16 September 2009; published 11 January 2010)

\begin{abstract}
We report on conductance measurements in carbon nanotube based double quantum dots connected to two normal electrodes and a central superconducting finger. By operating our devices as beam splitters, we provide evidence for crossed Andreev reflections tunable in situ. This opens an avenue to more sophisticated quantum opticslike experiments with spin entangled electrons.
\end{abstract}

DOI: 10.1103/PhysRevLett.104.026801

PACS numbers: 73.23. $-\mathrm{b}, 73.63 . \mathrm{Fg}$

Quantum optics has been an important source of inspiration for many recent experiments in nanoscale electric circuits [1,2]. One of the basic goals is the generation of entangled electronic states in solid state systems. Superconductors have been suggested as a natural source of spin entanglement, due to the singlet pairing state of Cooper pairs. One important building block required for the implementation of entanglement experiments using superconductors is a Cooper-pair beam splitter which should split the singlet state into two different electronic orbitals $[3,4]$.

The basic mechanism for converting Cooper pairs into quasiparticles is the Andreev reflection in which an originally quantum coherent electron pair in the singlet spin state is produced at an interface between a superconductor and a normal conductor. Conventional Andreev reflections (AR) are local and cannot readily be used to create bipartite states [5,6]. It has been suggested to make use of electronelectron interactions [6-13], spin filtering [14] or anomalous scattering in graphene [15] to promote Cooper-pair splitting, i.e., the crossed Andreev reflection (CAR) process.

In this Letter, we show that Coulomb interactions as well as size quantization can favor the CAR processes in carbon nanotubes. We use a double quantum dot geometry where the nanotube is connected to two normal electrodes and a central superconducting finger. By operating our device as a beam splitter (i.e., biasing the central superconducting electrode), we find that there is a finite current flowing from the superconducting electrode to the left $(L)$ arm and the right $(R)$ arm for a bias voltage smaller than the energy gap of the superconductor, which demonstrates Cooperpair injection. This subgap current is enhanced when we tune the device to the degeneracy points of the double dot with the help of capacitively coupled side gate electrodes. This enhancement together with the dependence of the asymmetry of transport in the superconducting state with respect to the normal state provide evidence for crossed Andreev reflections tunable in situ, in contrast to the weakly interacting metallic case $[16,17]$. These conclu- sions are supported by theoretical calculations based on a straightforward modeling of the device.

We use chemical vapor deposition to fabricate our single wall carbon nanotubes (SWNTs), which are localized with respect to Au alignment markers by scanning electron microscopy (SEM). A SEM picture of a typical device is shown in Fig. 1(a). We fabricate our devices using standard $e$-beam lithography and thin film deposition techniques.

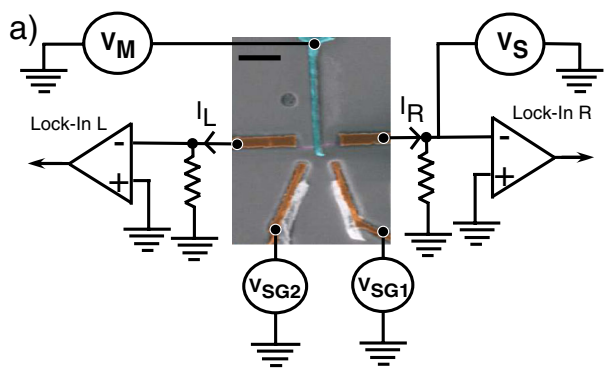

b)

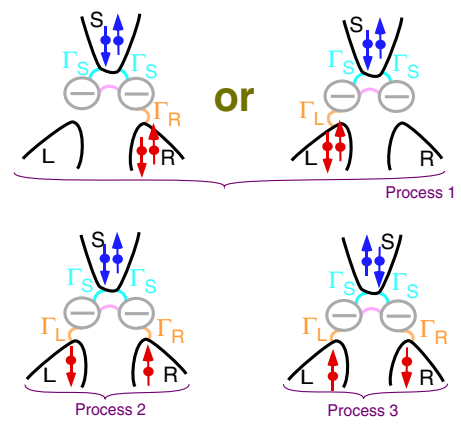

FIG. 1 (color online). (a) SEM image of a typical Cooper-pair splitter device in false colors with the two biasing schemes sketched. The bar is $1 \mu \mathrm{m}$. A central superconducting electrode is connected to two quantum dots engineered in the same single wall carbon nanotube (in purple [lightest gray]) which bridges between electrodes $L$ and $R$. (b) The elementary processes which carry current in the superconducting $(S)$ state. In addition to the conventional local Andreev reflection process, the crossed Andreev reflection can occur in which a Cooper pair is split in the two quantum dots. The relative probability of each of these processes can be inferred from the topology of the beam splitter. 
We deposit the normal and the superconducting contact in one fabrication step using shadow evaporation techniques. The normal contacts consist of $5 \mathrm{~nm}$ of titanium followed by $50 \mathrm{~nm}$ of $\mathrm{Au}$ or of $70 \mathrm{~nm}$ of Pd. The central superconducting electrode, $80 \mathrm{~nm}-100 \mathrm{~nm}$ wide, is a $\mathrm{Al}(100 \mathrm{~nm}) / \mathrm{Pd}(3 \mathrm{~nm})$ bilayer. Such a method allows us to achieve contact resistances as low as $30 \mathrm{kOhm}$ between the normal and superconducting reservoir. In addition to the highly doped $\mathrm{Si}$ substrate with $500 \mathrm{~nm} \mathrm{SiO}_{2}$ which is used as a global back gate, we implement two side gates whose voltage $V_{\mathrm{SG} 1}$ and $V_{\mathrm{SG} 2}$ can be tuned to control the two different parts defined by the central superconducting electrode. The spacing between the two normal contacts is between $600 \mathrm{~nm}$ and $1.2 \mu \mathrm{m}$. All the measurements presented in this Letter have been carried out in a dilution refrigerator with a base temperature of $80 \mathrm{mK}$, on one particular sample which fulfilled the (stringent) requirements of double dot spectroscopy and high enough coupling to the superconducting electrode. The currents flowing through the different arms of the beam splitter are measured via the voltage drop across two $2 \mathrm{k} \Omega$ resistors placed in series with the device as shown in Fig. 1(a).

For characterization, we first operate the device as a series double quantum dot by setting $V_{M}=0$ and $V_{S} \neq 0$ using the bias scheme shown in Fig. 1(a). Figure 2(a) displays the color scale plot of linear conductance $G_{L}=$ $d I_{L} / d V_{S}$ from the right $(R)$ to the left $(L)$ arm of the device as a function of side gate voltage $2 V_{\mathrm{SG} 2}$ and side gate

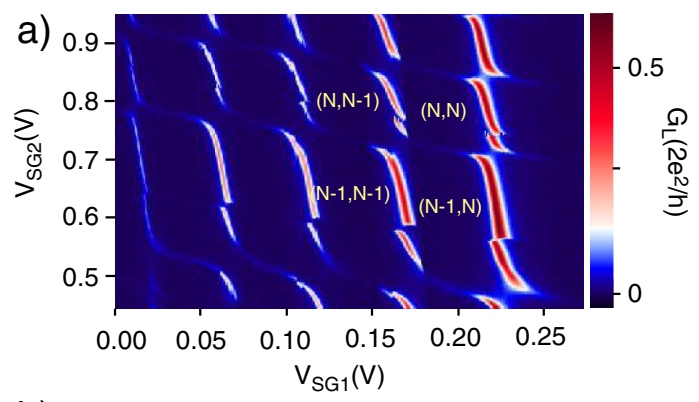

b)

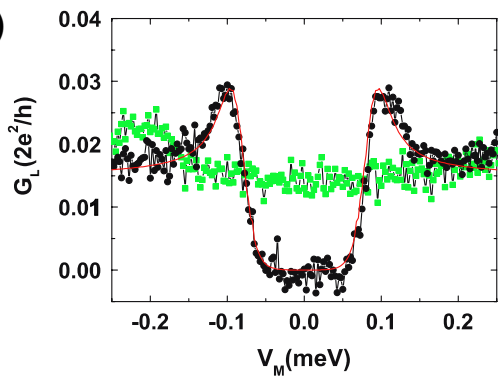

FIG. 2 (color online). (a) Color scale plot of the differential conductance $G_{L}$ as a function of side gate voltage $1 V_{\mathrm{SG} 1}$ and side gate voltage $2 V_{\mathrm{SG} 2}$. The dc bias voltage is zero here and the ac modulation $1.8 \mu \mathrm{V}$. (b) Differential conductance (black circles with lines) measured out of resonance in the middle injection scheme at $100 \mathrm{mK}$ and $0 \mathrm{mT}$. In green (light gray) squares, the differential conductance at $44.5 \mathrm{mT}$. In red (medium gray) solid lines, the BCS fit which yields an energy gap $\Delta$ of $85 \mu \mathrm{eV}$ and an electronic temperature of $100 \mathrm{mK}$. voltage $1 V_{\mathrm{SG} 1}$. The characteristic "honeycomb" stability diagram of a double quantum dot with rather regularly spaced avoided crossings is observed.

For the remainder of the Letter, we operate the device as a beam splitter by setting $V_{M} \neq 0$ and $V_{S}=0$. The differential conductance corresponding to the left $(L)$ arm and to the right $(R)$ arm of the beam splitter have qualitatively the same dependence as a function of $V_{M}, V_{\mathrm{SG} 1}$ or $V_{\mathrm{SG} 2}$. If $V_{\mathrm{SG} 1}$ and $V_{\mathrm{SG} 2}$ are tuned out of resonance, one typically measures a $G_{L}=d I_{L} / d V_{M}$ shown in Fig. 2(b). This demonstrates tunneling into a superconductor; i.e., the current is strongly suppressed for a bias voltage below the energy gap $\Delta$. Such a feature has been used very recently to probe the quasiparticle relaxation time in SWNTs [18]. A fitting to the thermally smeared BCS density of states gives $\Delta=$ $85 \mu \mathrm{eV}$ and an electronic temperature of $100 \mathrm{mK}$. Therefore, for $V_{M}<85 \mu \mathrm{V}$, one can only inject Cooper pairs. Note that the energy gap has a reduced value with respect to pure $\mathrm{Al}$ since it corresponds to the minigap of the $\mathrm{Al} / \mathrm{Pd}$ bilayer [19]. As shown in Fig. 2(b) in green (light gray) squares, the BCS gap does not appear if a magnetic field of $44.5 \mathrm{mT}$ is applied perpendicularly to the axis of the superconducting finger. This allows us to define the normal $(N)$ state of the beam splitter for which we apply a field of $89 \mathrm{mT}$ in order to be sure that superconductivity of the $\mathrm{Al} / \mathrm{Pd}$ slab is absent. The superconducting $(S)$ state is obtained for $0 \mathrm{mT}$.

The color scale plot of Fig. 3(a) displays $G_{L}$ for $V_{M}=$ $40 \mu \mathrm{eV}$ as a function of $V_{\mathrm{SG} 1}$ and $V_{\mathrm{SG} 2}$ for a specific anticrossing (AC1) in the $S$ state. In contrast to the off resonance case shown in Fig. 2(b), a relatively high subgap current $I_{S} \approx 0.1 I_{N}$ can flow into or from the superconductor even though $V_{M}$ is smaller than the energy gap at $\mathrm{AC} 1$. The presence of a subgap current can only be understood if Andreev reflections are taking place at resonance. In order to characterize the type of Andreev process occurring near the anticrossing, we show in Fig. 3(b) the variations of $G_{L}=d I_{L} / d V_{M}$ and $G_{R}=d I_{R} / d V_{M}$ measured simultaneously along the yellow arrow of the color scale plot in Fig. 3(a). In the $N$ state (black solid lines), $G_{L}$ and $G_{R}$ display two peaks corresponding to the bonding or antibonding states of the double dot. Because of an asymmetry of the coupling of each dot to the $L(R)$ reservoirs, the height of each doublet of the peaks is different. In the $S$ state represented in red (medium gray) solid lines, the peak height is reduced, but there is still a finite conductance for the $L(R)$ arm which follows essentially the resonances observed in the $N$ state. A similar feature is shown in Fig. 3(c) for another anticrossing (AC2) for which the asymmetry between $G_{L}$ and $G_{R}$ is slightly different. Note that the curves in the $N$ state have been scaled down by $1 / 3$ for the sake of clarity.

The elementary processes which contribute to the subgap current in our double quantum dot setup at the degeneracy point are schematically sketched in Fig. 1(b). The tunnel rates to the $S, L$, and $R$ contacts are respectively $\Gamma_{S}$, $\Gamma_{L}$, and $\Gamma_{R}$. The initial state in the superconductor $S$ is 

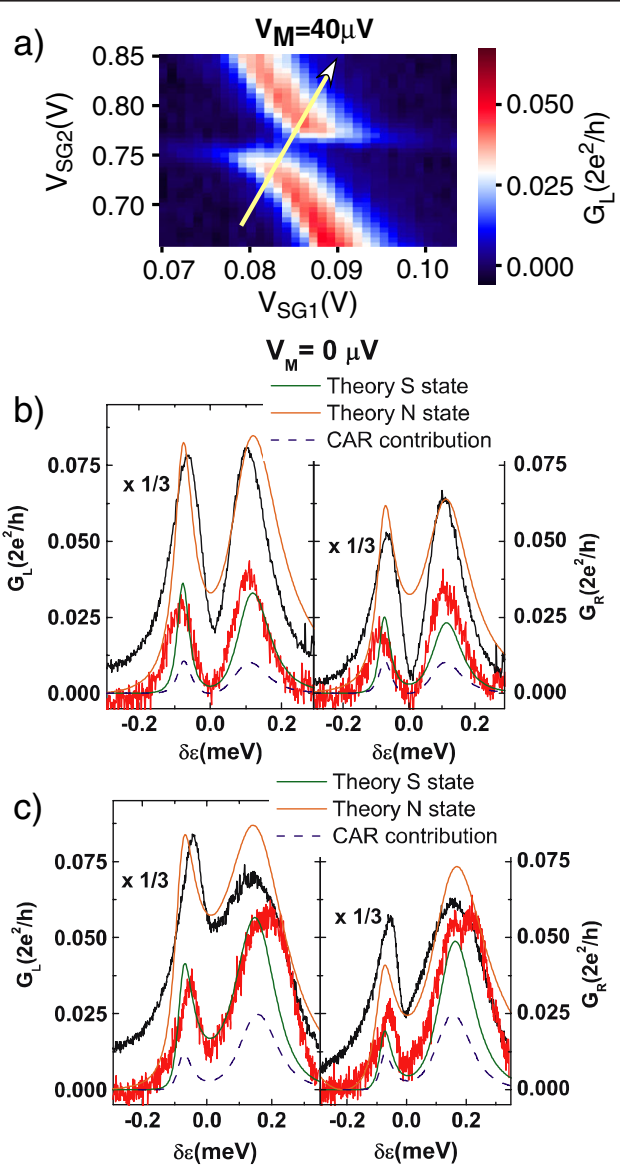

FIG. 3 (color online). (a) Focus on a specific region for the differential conductance $G_{L}$ as a function of $V_{\mathrm{SG} 1}$ and $V_{\mathrm{SG} 2}$ (AC1). The yellow arrow indicates the direction in which the linear scan of panel (b) has been taken. (b) Measurements of the conductance in the normal state (black solid lines) and in the superconducting state (red [medium gray] solid lines) for $G_{R}$ and $G_{L}$ along the direction of the yellow arrow. For the sake of clarity, the $N$ state conductances have been multiplied by $1 / 3$. The model calculations are in green [dark gray] solid lines for the $S$ state and in orange [light gray] solid lines for the $N$ state. In blue dashed lines, the CAR probability. (c) Similar graph as in (b) for anticrossing 2 (AC2).

represented in blue (dark gray) and the final state in the normal metals $N$, in red (medium gray). In process 1 , two particles are transferred to the same reservoir and the corresponding probability is proportional to $\Gamma_{L, R}^{2}$. In processes 2 and 3, which are equivalent, one particle is transferred to each reservoir. The corresponding probability is therefore proportional to $\Gamma_{L} \Gamma_{R}$. While the beam splitter geometry imposes the above general form for the Andreev tunneling probabilities, our theory (which we discuss in detail in [20]) allows to give an absolute value of the proportionality constants of the AR and the CAR, by accounting for higher order processes. Qualitatively, the enhancement of the CAR processes close to resonance results from the degeneracy of the electron states on the two dots, which allows the simultaneous tunneling of two electrons.
From the anticrossing of Fig. 3(a) and the full stability diagram of Fig. 2(a), we determine all the important parameters of the double dot using nonlinear transport similarly to what is done in Ref. [21], for example. The corresponding plots obtained from our theory at $T=0$ are given in green (light gray) solid lines for the $S$ state and in an orange (medium gray) solid line for the $N$ state for two anticrossings $\mathrm{AC} 1$ and $\mathrm{AC} 2$. Each anticrossing is characterized by the set of parameters $\left\{U_{L}, U_{R}, \Gamma_{12}, \Gamma_{L}\right.$, $\left.\Gamma_{R}, \Gamma_{S L}, \Gamma_{S R}\right\}, U_{L, R}$ being the on site charging energy, $\Gamma_{12}$ the coupling between the two dots, $\Gamma_{L}, \Gamma_{R}$ the coupling of each dot to its reservoir and $\Gamma_{S L}, \Gamma_{S R}$ the coupling of the superconductor to each dot. Although not all of these parameters can be determined independently, the fitting establishes the following hierarchy of energy scales: $U_{L, R} \approx 1 \mathrm{meV}, \Gamma_{12} \approx 100 \mu \mathrm{eV}, \Gamma_{L}, \Gamma_{R} \approx 100 \mu \mathrm{eV}$ and $\Gamma_{S L}, \Gamma_{S R} \approx 10 \mu \mathrm{eV}$. For both arms and both anticrossings, the calculated current in the superconducting state is in good agreement with our experimental findings, which implies an important contribution from CAR processes, as shown by the dashed lines in Figs. 3(b) and 3(c). For $\mathrm{AC} 1$, for example, the fit allows us to deduce a contribution of split Cooper pairs up to $35 \%$ for $G_{L}$ and $55 \%$ for $G_{R}$ from our transport model.

Now, we exploit the asymmetry of transport between the $L$ and $R$ arms to provide further evidence for the Cooperpair splitting. In the $S$ state, it is defined as $\alpha_{S}=G_{L} / G_{R}$ at $0 \mathrm{mT}$, and in the $N$ state, as $\alpha_{N}=G_{L} / G_{R}$ at $89 \mathrm{mT}$, both taken at resonance. One can already anticipate from the elementary processes of Fig. 1(b) that the presence or the absence of processes 2 and 3 should be observable directly from the dependence of $\alpha_{S}$ with respect to $\alpha_{N}$. At the degeneracy point, both the local and nonlocal Andreev processes acquire a Breit-Wigner-like form at resonance (see [20]). In the limit $\Gamma_{12} \gg \Gamma_{L, R} \gg \Gamma_{S}$, we obtain for the conductance $G_{L(R)}$ :

$$
G_{L(R)}=\frac{4 e^{2}}{h} \frac{16 \tilde{\Gamma}_{S}^{2}}{\left[\Gamma_{L}+\Gamma_{R}\right]^{4}}\left[\Gamma_{L(R)}^{2}+\Gamma_{L} \Gamma_{R}\right],
$$

where $\tilde{\Gamma}_{S}$ is the tunnel rate to the superconductor renormalized by Coulomb interactions. The presence of the crossed term $\Gamma_{L} \Gamma_{R}$ in Eq. (1) implies that:

$$
\alpha_{S}=\frac{\Gamma_{L}^{2}+\Gamma_{L} \Gamma_{R}}{\Gamma_{R}^{2}+\Gamma_{L} \Gamma_{R}}=\frac{\Gamma_{L}}{\Gamma_{R}}=\alpha_{N}
$$

Away from the degeneracy points, when one of the dots is blocked, only local AR are present and the term proportional to $\Gamma_{L} \Gamma_{R}$ in Eq. (1) is absent. In this case, $\alpha_{S}$ is just the square of $\alpha_{N}$. The bottom panel of Fig. 4 displays $\alpha_{S}$ versus $\alpha_{N}$ for all the anticrossings which we have measured, in red squares, and resonances away from the anticrossings, in black stars. The 31 red squares correspond to 7 different anticrossings. The error bars correspond to the systematic error made when determining $\alpha_{S}$. The black stars are obtained when one of the two dots is Coulomb blockaded (single resonance case). Within the error bars, 


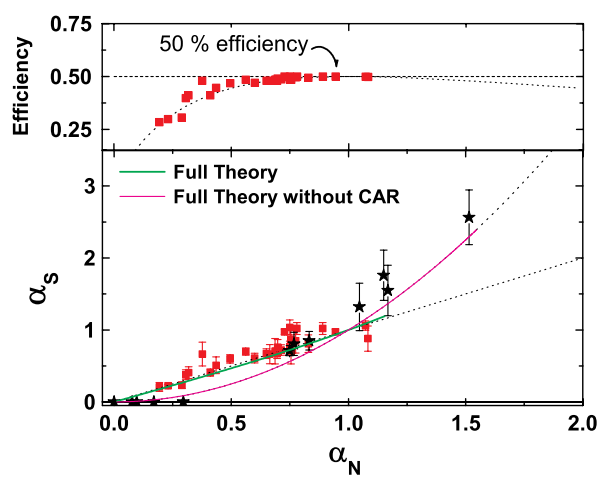

FIG. 4 (color online). Bottom panel: Asymmetry in the superconducting state versus asymmetry in the normal state for 31 out of the 35 anticrossings studied (red filled squares) and 11 resonance peaks away from the anticrossings (black stars). The dashed curve corresponds to $\alpha_{S}=\alpha_{N}^{2}$. The dashed line corresponds to $\alpha_{S}=\alpha_{N}$. The green [medium gray] solid line is the full theory (see[20]) and the pink [light gray] solid line is the full theory without the CAR process. Top panel: Efficiency of the Cooper-pair splitting for the device as deduced from the values of $\alpha_{S}$ (see text). In dashed lines, the theoretical curve.

they fall onto the dashed curve which corresponds to the universal parabola $\alpha_{S}=\alpha_{N}^{2}$ as expected. The red square points fall onto the universal dashed line $\alpha_{S}=\alpha_{N}$ [22]. The observed contrast between the quadratic behavior followed by the black stars and the linear behavior followed by the red squares proves the Cooper-pair splitting action of our device. Note that the full theory [i.e., without the approximations leading to Eq. (1)] predicts a dependence of $\alpha_{S}$ versus $\alpha_{N}$ which is marginally different from these behaviors in our parameter range as shown by the green (medium gray) and pink (light gray) curves in Fig. 4. The universal linear behavior arising from the Cooper-pair splitting is therefore a very useful diagnosis tool for a wide range of parameters of our beam splitter. The efficiency of the beam splitter can be defined as the ratio $2 T_{\mathrm{CAR}} /\left(A R_{L}+\right.$ $\left.A R_{R}+2 T_{\mathrm{CAR}}\right) \approx 2 /\left(2+1 / \alpha_{S}+\alpha_{S}\right)$ of the split current to the total current. It is displayed in the top panel of Fig. 4. For a $\alpha_{N}$ ranging from 0.5 to 1 , the efficiency of Cooperpair splitting is close to $50 \%$, showing that almost half of the Cooper pairs flowing out of the superconducting finger are split at the SWNT.

In conclusion, we have shown that carbon nanotube double quantum dots can be used as tunable Cooper-pair beam splitters. The specific advantage of beam splitters based on double quantum dots is the possibility of further processing the electron states, e.g., by spin filtering for EPR type experiments.

We thank A. Cottet for a critical reading of the manuscript and illuminating discussions. We thank The Mesosocopics group of LPA, The Quantronics Group and J. Siewert for assistance and fruitful discussions. This work is supported by the SFB 689 of the Deutsche Forschungsgemeinschaft, the ANR-05-NANO-028 con- tract, the ANR-07-NANO-011-004 contract, the EU Contract No. FP6-IST-021285-2, the C'Nano Ile de France contract SPINMOL, the Spanish MICINN under Contract No. FIS2008-04209 and the DFH-UFA and DAAD mobility grants.

Note added.-During the course of writing this Letter, we became aware of a similar study using InAs nanowires by Hofstetter et al. [23].

*To whom correspondence should be addressed: kontos@lpa.ens.fr

[1] G. Fève, A. Mahé, and J.-M. Berroir et al., Science 316, 1169 (2007).

[2] A. Wallraff, D. I. Schuster, and A. Blais et al., Nature (London) 431, 162 (2004).

[3] N. M. Chtchelkatchev, G. Blatter, G. B.Lesovik, and T. Martin, Phys. Rev. B 66, 161320 (2002).

[4] P. Samuelsson, E. V. Sukhorukov, and M. Büttiker, Phys. Rev. Lett. 91, 157002 (2003).

[5] T. Martin, Phys. Lett. A 220, 137 (1996).

[6] P. Recher, E. V. Sukhorukov, and D. Loss, Phys. Rev. B 63, 165314 (2001).

[7] G. B. Lesovik, T. Martin, and G. Blatter, Eur. Phys. J. B 24, 287 (2001).

[8] M.-S. Choi, C. Bruder, and D. Loss, Phys. Rev. B 62, 13569 (2000).

[9] P. Recher and D. Loss, Phys. Rev. B 65, 165327 (2002).

[10] C. Bena, S. Vishveshwara, L. Balents, and M. P. A. Fisher, Phys. Rev. Lett. 89, 037901 (2002).

[11] V. Bouchiat, N. Chtchelkatchev, and D. Feinberg et al., Nanotechnology 14, 77 (2003).

[12] A. Levy Yeyati, F. S. Bergeret, A. Martín-Rodero, and T. M. Klapwijk, Nature Phys. 3, 455 (2007).

[13] E. Dupont and K. Le Hur, Phys. Rev. B 73, 045325 (2006).

[14] G. Deutscher and D. Feinberg, Appl. Phys. Lett. 76, 487 (2000).

[15] J. Cayssol, Phys. Rev. Lett. 100, 147001 (2008).

[16] D. Beckmann, H. B. Weber, and H. v. Löhneysen, Phys. Rev. Lett. 93, 197003 (2004).

[17] S. Russo, M. Kroug, T. M. Klapwijk, and A. F. Morpurgo, Phys. Rev. Lett. 95, 027002 (2005).

[18] Y.-F. Chen, T. Dirks, G. Al-Zoubi, N. O. Birge, and N. Mason, Phys. Rev. Lett. 102, 036804 (2009).

[19] T. Kontos, M. Aprili, J. Lesueur, X. Grison, and L. Dumoulin, Phys. Rev. Lett. 93, 137001 (2004).

[20] See supplementary material at http://link.aps.org/supplemental/10.1103/PhysRevLett.104.026801.

[21] M. Gräber, W. A. Coish, and C. Hoffmann et al., Phys. Rev. B 74, 075427 (2006).

[22] As we discuss in the EPAPS [20] we do not include in Fig. 4 points corresponding to 2 anticrossings for which the condition $\Gamma_{L, R} \gg \Gamma_{S}$ did not apply and which were anomalously sensitive to detuning. It should be mentioned, however, that they can be fully accounted by the full theory, as shown in the EPAPS.

[23] L. Hofstetter, S. Csonka, J. Nygard, and C. Schönenberger, Nature (London) 461, 960 (2009). 